\title{
Human Fibroblast-derived Multi-peptide Factors and the Use of Energy-delivering Devices in Asian Patients
}

\author{
Sang Bum Suh ${ }^{1, *}$ \\ Keun Jae $\mathrm{Ahn}^{2, *}$ \\ Hye Jin Chung ${ }^{3}$ \\ Ji Youn Suh ${ }^{1}$ \\ Sung Bin $\mathrm{Cho}^{4}$
}

\author{
${ }^{1} R \& D$ Center, BNV Biolab, Seoul, Korea \\ ${ }^{2}$ Department of Science Education, Jeju National \\ University, Jeju, Korea \\ ${ }^{3}$ Department of Dermatology, Beth Israel \\ Deaconess Medical Center, Boston, MA, USA \\ ${ }^{4}$ Yonsei Seran Dermatology and Laser Clinic, \\ Seoul, Korea
}

Received May 24, 2020

Accepted May 25, 2020

\author{
Correspondence \\ Sung Bin Cho \\ Yonsei Seran Dermatology and Laser Clinic, 224 \\ Siheung-daero, Seoul 08628, Korea \\ Tel.: +82-2-2135-1375 \\ Fax: $+82-70-8250-1375$ \\ E-mail: drsbcholagmail.com \\ *The first two authors equally contributed to this \\ work.
}

(C) Korean Society for Laser Medicine and Surgery

(c) This is an open access article distributed under the terms of the Creative Commons Attribution NonCommercial License (http://creativecommons.org/ licenses/by-nc/4.0) which permits unrestricted noncommercial use, distribution, and reproduction in any medium, provided the original work is properly cited.
Human fibroblast-derived multi-peptide factors (MPFs) have been used during treatments with energy-delivering modalities to enhance energyinduced tissue reactions. Human fibroblast-derived MPFs, which include a range of growth factors and chemoattractive factors, activate and recruit fibroblasts and endothelial cells, as well as promote extracellular matrix deposition, all of which are crucial to wound repair. Interestingly, fibroblasts from different species or anatomical sites exhibit distinct transcriptional properties with high heterogeneity. In addition, the patterns of MPF secretion can differ under a range of experimental conditions. Therefore, the use of allogeneic fibroblasts and proper cultivation thereof are necessary to obtain MPFs that can enhance the epithelial-mesenchymal interactions during wound repair. Moreover, energy-delivering devices should be selected according to evidence demonstrating their therapeutic efficacy and safety on a pathological skin condition and the major target skin layers. This paper reviewed the histologic patterns of post-treatment tissue reactions elicited by several energy sources, including non-ablative and ablative fractional lasers, intense focused ultrasound, non-invasive and invasive radiofrequency, picosecond-domain lasers, and argon and nitrogen plasma. The possible role of the immediate application of human fibroblast-derived MPFs during wound repair was proposed.

\section{Key words}

Fibroblast-derived multi-peptide factors; Energy-delivering system; Radiofrequency; Fractional laser; Ultrasound; Picosecond-domain laser; Plasma; Argon; Nitrogen 


\section{IMMEDIATE/LATE SKIN REACTIONS FOR VARIOUS ENERGY-DELIVERING DEVICES}

\section{Non-ablative and ablative fractional lasers}

Non-ablative fractional lasers (NAFLs) and ablative fractional lasers (AFLs) are frequently used modalities for skin rejuvenation or for treating atrophic skin lesions or hair loss. ${ }^{1-3}$ Commonly used NAFL devices include 1,540or 1,550-nm erbium-glass NAFL and 1,927-nm thulium NAFL. A 1,540- or 1,550-nm erbium-glass NAFL generates immediate fractionated thermal tissue reactions of vertical coagulation columns along the epidermis, upper dermis, and follicular epithelium all the while preserving the integrity of the stratum corneum. ${ }^{1,2}$ Further, NAFL treatments elicit the remodeling of collagen in scars into mature interwoven bundles. 'High-fluenced NAFL treatments, however, are associated with a risk of posttreatment granuloma formation.

Thulium laser pulses at a wavelength of 1,927 nm exhibit an absorption coefficient to water higher than that for 1,540- or 1,550-nm erbium-glass laser pulses, but much lower than that for $10,600-n m$ carbon dioxide $\left(\mathrm{CO}_{2}\right)$ laser pulses. ${ }^{4}$ The immediate tissue reactions of 1,927-nm thulium NAFLs present as inverted cone-shaped zones of thermal coagulation along the epidermis and upper dermis (Fig. 1), wherein the integrity of the epidermis and follicular epithelium are preserved. ${ }^{3}$ Moreover, environmental scanning electron microscopic study has revealed that low-energy thulium NAFL treatments generate multiple, heterogeneous, round micropores within the zones of laser-induced thermal coagulation with a mean estimated diameter of $6.7 \pm 4.1 \mu \mathrm{m}{ }^{3}$ Late thulium NAFLinduced tissue reactions exhibit notably thickened epidermis and increased dermal collagen fibers and fibroblasts without remarkable scar tissue formation. ${ }^{3}$

Commonly used AFL devices include 2,940-nm erbium (Er):yttrium-aluminum-garnet (YAG) and 10,600-nm CO lasers. Two distinctive AFL delivery systems are generally used: 1) fractional high-fluenced laser pulses, which generate narrow and deep ablative and coagulative zones along the epidermis and the upper and mid dermis, with surrounding uninjured skin at a percent coverage of less than 30\% usually, and 2) fractional low-fluenced laser pulses, which produce wide and shallow ablative and coagulative zones limited to the upper most epidermis at a percent overage over $100 \%{ }^{5}$ Most ablative and coagulative laser-induced thermal reactions generated by highfluenced AFL treatments in the epidermis and dermis heal within 5 days, leaving microscopic clefts in the dermoepidermal junction (Fig. 2). ${ }^{6}$ In one study, human specimens obtained at Day 7, 10, 14, and 21 after treatment with high-fluenced AFL exhibited marked thickening of the epidermis and upper dermis without noticeable scar formation. ${ }^{6}$ Moreover, although thermal coagulation and ablation areas were limited to the uppermost epidermis immediately after low-fluenced AFL resurfacing treat-
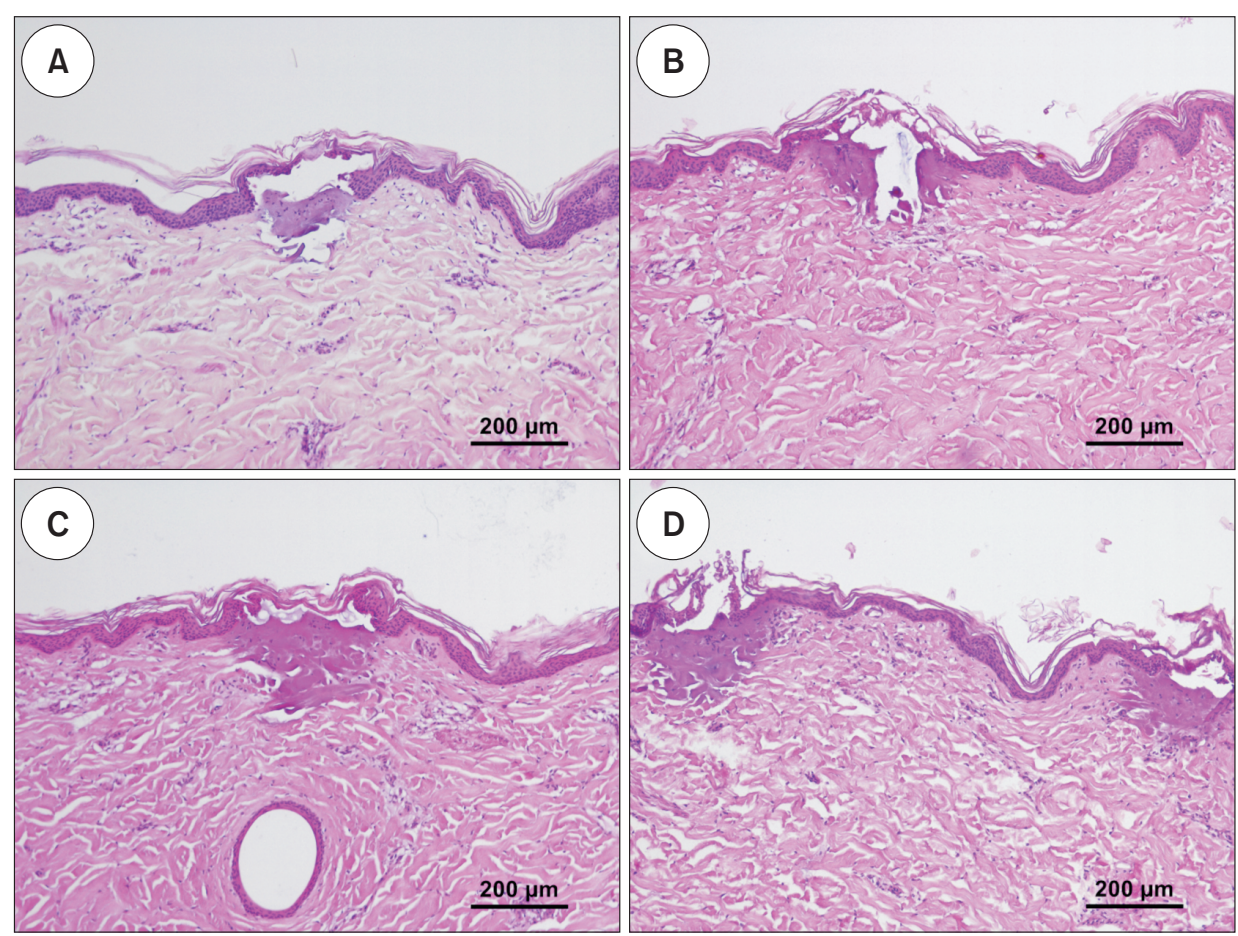

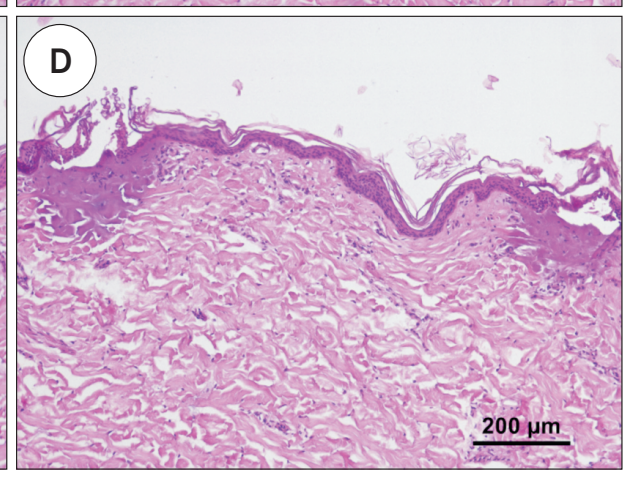

Fig. 1. Immediate skin reactions after 1,927-nm fractional laser treatment. Inverted cone-shaped zones of thermal coagulation along the epidermis and upper dermis of ex vivo female minipig skin after a single pass with a $1,927-\mathrm{nm}$ fractional laser (LaseMD ULTRA ${ }^{\mathrm{TM}}$; Lutronic Corp., Goyang, Korea) using LaseMD Ultra ${ }^{\mathrm{TM}} \mathrm{C} 1$ tip (Lutronic Corp.) at the settings of (A) $5 \mathrm{~W}$ and $10 \mathrm{~mJ}$, (B) $5 \mathrm{~W}$ and 15 $\mathrm{mJ},(\mathrm{C}) 5 \mathrm{~W}$ and $20 \mathrm{~mJ}$, and (D) $20 \mathrm{~W}$ and $20 \mathrm{~mJ}$. The integrity of the epidermis is microscopically preserved. Hematoxylin and eosin stain, original magnification $\times 100$. 

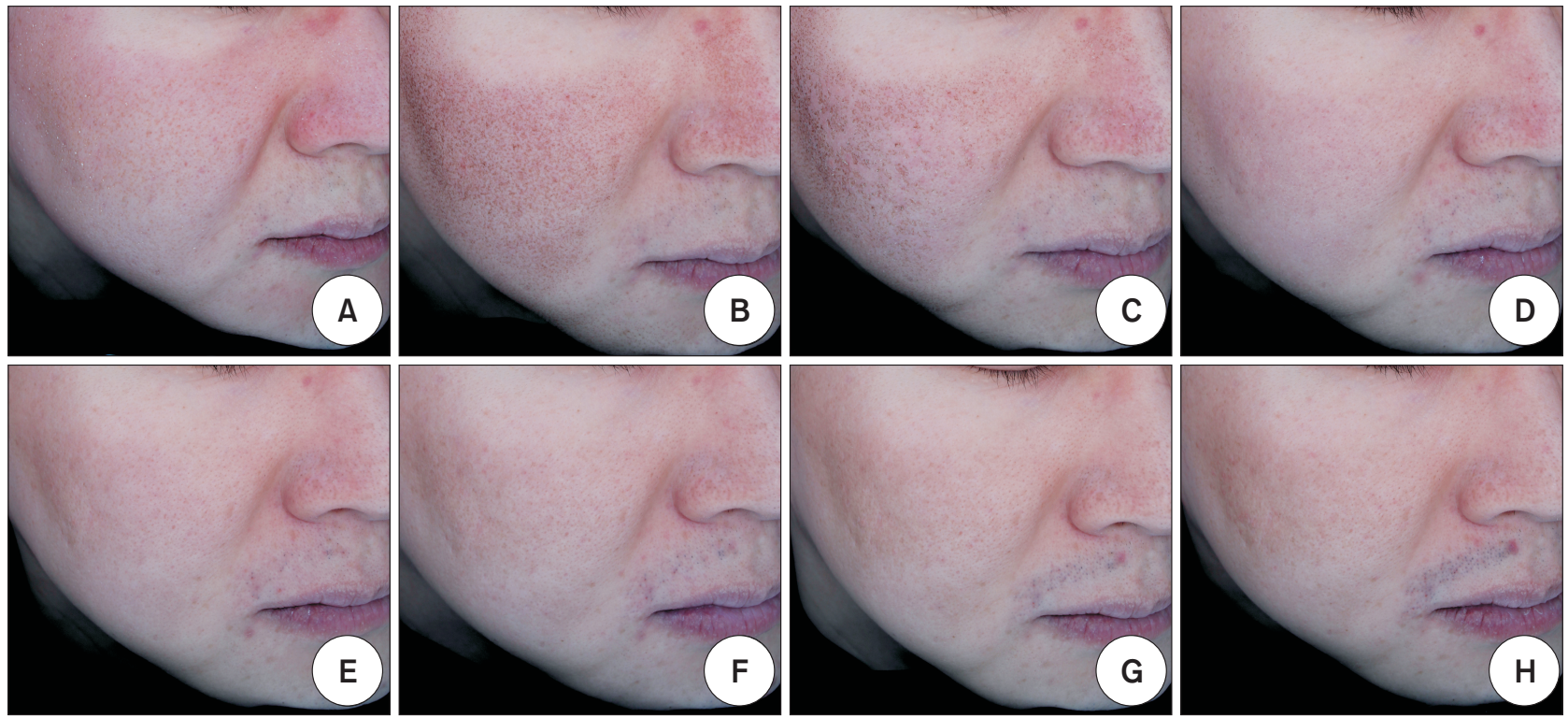

Fig. 2. In vivo human skin reaction after a hybrid fractional laser treatment. Clinical course of wound repair after the hybrid non-ablative 1,470-nm diode and ablative 2,940-nm erbium (Er):yttrium aluminum garnet (YAG) fractional laser (Halo ${ }^{\mathrm{TM}}$ Pro; Sciton, Palo Alto, CA, USA) treatment. Treatment settings with the non-ablative 1,470-nm diode laser include a penetration depth of $325 \mu \mathrm{m}$ and a percent coverage of $10 \%$ and those with the ablative 2,940-nm Er:YAG laser include a penetration depth of $20 \mu \mathrm{m}$ and a percent coverage of 10\%. Photographs were taken (A) immediately, (B) 1 day, (C) 3 days, (D) 5 days, (E) 1 week, (F) 2 weeks, (G) 3 weeks, and (H) 4 weeks after treatment. Polarized light exposure, right oblique view.

ments, those treatments induced cytokine and chemokine expression that effectively increased the production of dermal type I and type III procollagen. ${ }^{5}$

\section{Intense focused ultrasound}

Ultrasound waves can be used to generate frictional heat in the skin by causing composite molecules to vibrate. ${ }^{7.8}$ When ultrasound energy is focused on cutaneous tissue, a well-defined focal zone of ultrasound-induced thermal injury, the size of which is usually $1-\mathrm{mm}^{3}$ or smaller, at pre-set penetration depths, particularly 1.5, 3.0 , or $4.5 \mathrm{~mm}$, can be generated, sparing the epidermis from excessive heat injury. ${ }^{7}$ Intense focused ultrasound (IFU) at high energy settings generates an "island and moat" pattern composed of islands of IFU-induced coagulative necrosis reaching irreversible cytotoxic temperatures of up to $60^{\circ} \mathrm{C}$ and moats of normal-looking, glycogen free cells. ${ }^{7}$

Fresh cadaveric skin studies have demonstrated that IFU treatments on the face and neck result in well-demarcated round to oval zones of coagulation and ablation in the dermis at a pre-set penetration depth of $1.5 \mathrm{~mm}$, in the lower dermis to upper subcutaneous fat tissue at 3.0 $\mathrm{mm}$, and in the lower subcutaneous fat tissue to fascia at $4.5 \mathrm{~mm} .{ }^{9.10}$ Depending on the device used, distinctive cylindrical columns of thermal coagulation can be found at pre-focal areas in the fresh cadaveric skin: ${ }^{10}$ pre-focal tissue coagulation reactions are expected to induce neocollagenesis and neoelastogenesis that results in skin rejuvenation and tightening. Research has indicated that these pre-focal tissue reactions originate from the reflection of ultrasound waves in the dermo-subcutaneous fat junction and are specific to histologic features of cadaveric skin, but not in vivo human skin. In vivo human skin specimens post-IFU treatment present with remarkable increases in collagen fibers in the mid and deep reticular dermis and elastic fibers in the deep reticular dermis. ${ }^{11}$

\section{Non-invasive and invasive radiofrequency Monopolar radiofrequency}

Radiofrequency (RF) energy, which can be emitted noninvasively or invasively via a monopolar or bipolar mode, generates electrothermal reactions in a target tissue. ${ }^{7,12,13}$ The electrical circuit formed with a monopolar RF system begins from the active electrode and propagate to the grounded electrode. ${ }^{7}$ Non-invasive monopolar RF energy has been found to generate columns of thermal coagulation without desiccative tissue reactions along the epidermis and mid to lower dermis. ${ }^{10}$ Therein, the maximum thermal reaction is usually found in the mid to lower part of the coagulation column. ${ }^{10}$ Higher power settings have been found to elicit a greater degree of tissue coagulation, 
while longer conduction-time settings appear to generate deeper and wider coagulation areas. ${ }^{10}$

Invasive monopolar RF treatment at a low-energy setting using non-insulated penetrating electrodes generates well-demarcated, round to oval, coagulation zones at the tips of microneedles. ${ }^{14}$ The coagulation zones then propagate to the proximal parts of penetrating electrodes with increasing RF energy. ${ }^{14}$ The thickest areas of coagulated tissue are found around the tips of the electrodes. ${ }^{14}$ Therefore, even with non-insulted, penetrating microneedle electrodes, monopolar RF treatments can be used to coagulate targeted dermal tissue while avoiding unwanted thermal injury to the epidermis.

\section{Bipolar radiofrequency}

Bipolar RF systems generate electrothermal tissue reactions within and around an electrical circuit formed between active electrodes. ${ }^{15,16}$ The maximum depth of tissue reactions generated when using non-invasive, bipolar RF devices primarily depends on the distance between the electrodes. ${ }^{16}$ Although he depth of RF-induced tissue reactions is deeper with non-invasive monopolar RF systems, it can be more precisely regulated with noninvasive bipolar RF systems. ${ }^{16}$

Clinically, non-invasive bipolar RF devices have been found to uniformly heat target tissue to temperatures over $41-43^{\circ} \mathrm{C}$ for several minutes: a real-time system for measuring tissue impedance and temperature and immediate feedback regulation of RF delivery is required to uniformly heat target skin and to prevent possible side effects. ${ }^{17,18}$ Although immediate skin reactions of coagulation or ablation areas cannot be found, post-RF skin specimens exhibit significant increases in dermal collagen fibers and collagen bundle densities, but not elastin. ${ }^{18}$ Accordingly, the more uniform and longer exposure of non-invasive bipolar RF energy has been suggested to be effective at promoting neocollagenesis and collagen remodeling. ${ }^{17,18}$

Non-invasive bipolar RF energy can be used to reduce adipose tissue and to rejuvenate overlying skin. ${ }^{19}$ Immediate RF-induced tissue reactions in adipose tissue include shrunken and withered fat cell membranes, reduced fat cell size, elongated or flattened fat cells, and partially ruptured adipocytes. ${ }^{19}$ These RF-induced adipocyte changes have been suggested to be related with RF-induced apoptosis, but not necrosis. ${ }^{19}$ Furthermore, adjacent structures, including the epidermis, dermal collagen, vascular components, and nerve fibers, are well-preserved. ${ }^{19}$ Long-term effects include more pronounced adipose tissue changes and significantly reduced dermal thickness with more compact collagen, compared with untreated skin or post-RF immediate skin specimens. ${ }^{19}$

To non-invasively deliver bipolar RF energy to deeper parts of the skin that contains fat cells, a suction-coupled, real-time feedback RF system employing a vacuum to draw the tissue up between the electrodes can be used. ${ }^{19}$ Moreover, two types of RF pulses are emitted, comprising basic 1-MHz RF pulses and high-signal amplitude, ultrashort pulse duration RF pulses. The former uniformly heats the dermis and subcutaneous fat to induce neocollagenesis and skin rejuvenation. ${ }^{19-21}$ The latter high-signal amplitude RF elicits irreversible electroporation of adipocyte membranes, which ultimately results in cellular apoptosis. $^{19-21}$

For invasive bipolar RF systems, the penetration depths of electrodes can be controlled to regulate the depth of energy delivery (Fig. 3). 12,13,16 Previous research has outlined the patterns of invasive bipolar RF-induced thermal reactions in in vivo and ex vivo tissue models. ${ }^{12,13}$ Experimental settings with 2-MHz bipolar RF, "non-insulated" penetrating electrodes, and 25 microneedles in a $5 \times 5$ pattern elicited round to oval zones of tissue coagulation in the dermis that started from the tips of each electrode without thermal injury in the epidermis. ${ }^{12}$ With increasing RF conduction time, coagulation areas propagated along the entire length of the microneedle electrodes. ${ }^{12}$ Then, convergence thereof on neighboring coagulation columns became apparent starting from the distal and mid parts of the electrodes through the shortest pathway. ${ }^{12}$ In another experimental setting with 2-MHz bipolar RF, "insulated" penetrating electrodes, and 49 microneedles in a 7 × 7 pattern, well-demarcated zones of tissue coagulation confined to the tips of individual electrodes were generated. $^{13}$ Therein, larger areas of dermal tissue were coagulated at a deeper treatment depth and/or with longer RF conduction times, and greater degrees of RF-induced thermal injury were found at higher RF energy. ${ }^{13}$ Convergence among individual coagulation columns, however, was not found. ${ }^{13}$

Previous investigations analyzing the patterns of RF-induced tissue reactions faced a significant limitation in that the effects of RF-induced tissue desiccation upon further energy delivery to the target tissue could not be precisely evaluated. $^{12,13,22}$ This is because RF-induced tissue dehydration in the peri-electrode areas, as the temperature rises to around $100^{\circ} \mathrm{C}$, increases impedance that limits further RF energy delivery. ${ }^{22}$ Therefore, real-time feedback, power-controlling systems have been developed to minimize desiccative tissue injury and to potentially increase the efficacy of RF delivery. However, further controlled experimental studies should be followed to con- 

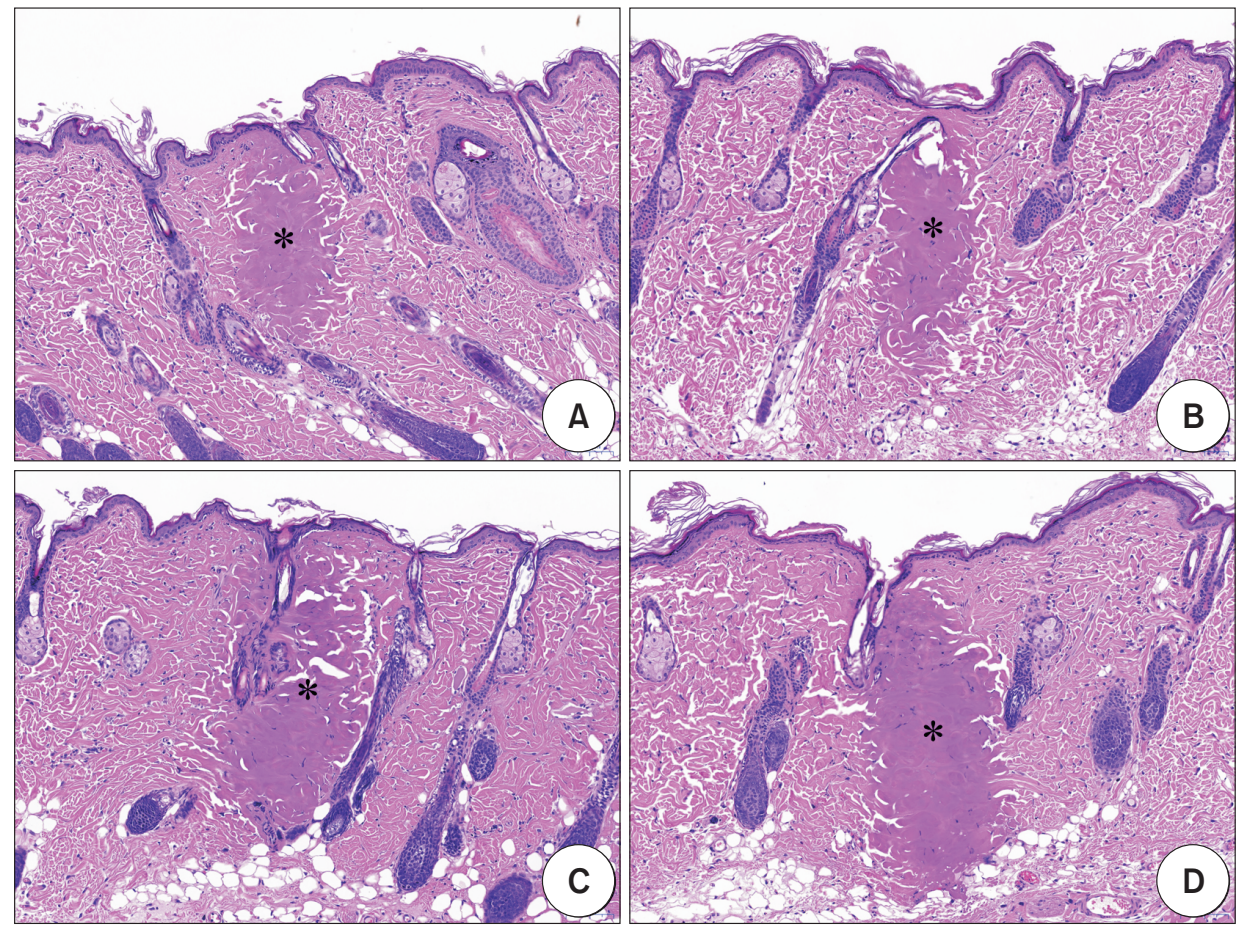

Fig. 3. In vivo rat skin reactions after invasive bipolar radiofrequency (RF) treatment. (A, B) Single-pass, invasive bipolar RF (Vivace ${ }^{\mathrm{TM}}$; Shenb Co., Ltd., Seoul, Korea) treatment using 36 insulated microneedles in a $6 \times 6$ pattern generates well-demarcated oval zones of tissue coagulation (asterisks) in the dermis of in vivo female Sprague-Dawley rat skin at a frequency of $1 \mathrm{MHz}$, a RF conduction time of $200 \mathrm{msec}$, a microneedle penetration depth of $2 \mathrm{~mm}$, and a power of (A) $50 \mathrm{~W}$ and (B) $70 \mathrm{~W}$. (C, D) Meanwhile, single-pass, invasive bipolar RF treatment using insulated microneedles generates larger areas of tissue coagulation (asterisks) in the dermis at a frequency of $1 \mathrm{MHz}$, a RF conduction time of $500 \mathrm{msec}$, and a power of $(\mathrm{C}) 50 \mathrm{~W}$ and (D) $70 \mathrm{~W}$. Hematoxylin and eosin stain, original magnification $\times 100$.

firm the effects of real-time feedback, power-controlling systems on RF-induced tissue reactions.

\section{Picosecond-domain lasers}

Optical pulses from picosecond-domain lasers generate greater photoacoustic effects on target chromophores than those from nanosecond-domain lasers. ${ }^{23-25}$ Picosecond laser-induced immediate tissue reactions exhibit more remarkable microscopic vacuolization in the epidermis and upper dermis at lower-fluence treatment settings than those achieved with nanosecond lasers. ${ }^{26}$ The degree of vacuolization is usually greater throughout the epidermis and extends deeper to the upper dermis with higher fluence settings. ${ }^{26}$ Moreover, a wavelength of $532 \mathrm{~nm}$, which has a greater absorption coefficient by both hemoglobin and melanin, elicits a greater tissue reaction, despite higher scatter loss, than a wavelength of $1,064 \mathrm{~nm}^{26}$

Fractionated optics for picosecond lasers, including a microlens-array (MLA) optic and a diffractive optical element (DOE), generate fractionated high-fluenced areas and surrounding low-fluenced background areas. ${ }^{23,26,27}$
Therein, non-invasive, non-ablative, fractional picosecond laser treatments produce thermally-initiated laserinduced optical breakdown (TI-LIOB) injuries in the epidermis and upper dermis that stimulate the production of cytokines, chemokines, and growth factors for skin rejuvenation in patients with atrophic scars, enlarged pores, and wrinkles. ${ }^{23,24} \mathrm{~A}$ previous ex vivo genotype-regulated pigmented micropig skin study describe the appearance of large cystic vacuoles in the epidermis and dermis with surrounding multiple microscopic vacuoles. ${ }^{26}$ As the distance between the micro-lens and the skin grew longer, the degree of tissue vacuolization became less, while the area of microscopic vacuolization became wider. ${ }^{26}$

The patterns of skin reactions for MLA-type, 532- and 1,064-nm, picosecond-domain, neodymium (Nd):YAG laser treatments in an ex vivo Asian skin specimen have been described. ${ }^{27}$ Therein, focal, large cystic vacuoles of TI-LIOB were found in the epidermis, dermo-epidermal junction, and upper papillary dermis at regular intervals with surrounding microscopic vacuoles in both 532- and 1,064-nm treatment settings (Fig. 4). ${ }^{27}$ Most of the large cystic TI-LIOB lesions in the epidermis presented CD31- 

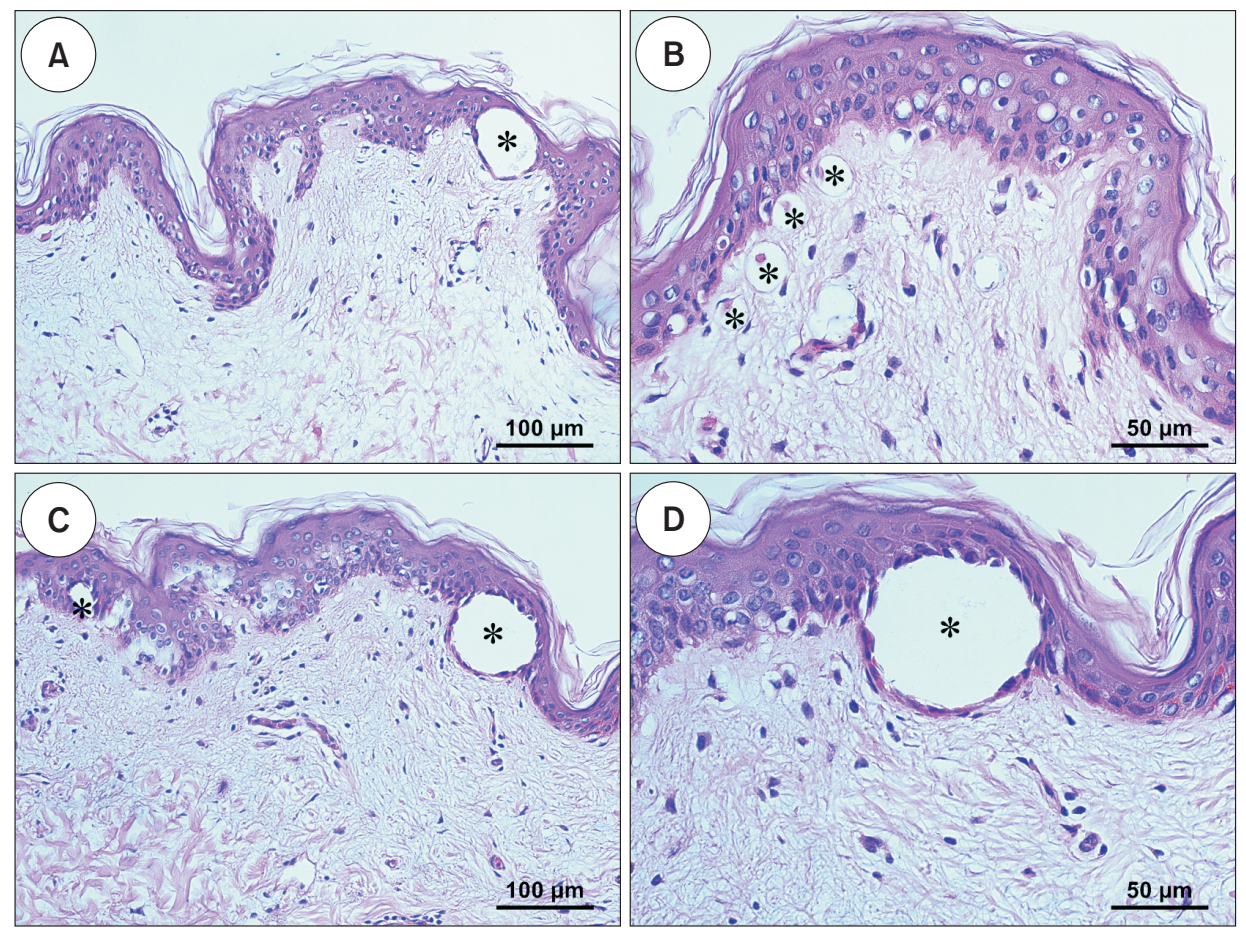

Fig. 4. Ex vivo human skin reactions after 532-nm and 1,064-nm microlens array (MLA)-type, picosecond laser treatment. (A, B) Single-pulse, 532-nm, picosecond laser (PicoPlus ${ }^{\circledR}$; Lutronic Corp.) treatment at a 6-mm spot size, a pulse duration of 450 picoseconds, a fluence of $1.0 \mathrm{~J} / \mathrm{cm}^{2}$, and a distance between the microlens and the skin of $48 \mathrm{~mm}$ generates cystic cavitation lesions (asterisks) and microscopic perinuclear vacuolar changes in the lower epidermis and papillary dermis in ex vivo human skin with Fitzpatrick skin type III. (C, D) Single-pulse, 1,064-nm, picosecond laser treatment at a 7-mm spot size, a pulse duration of 450 picoseconds, a fluence of $1.9 \mathrm{~J} / \mathrm{cm}^{2}$, and a distance between the microlens and the skin of $31 \mathrm{~mm}$ generates the fractionated appearance of cystic cavitation lesions (asterisks) and perinuclear vacuolar changes in the lower epidermis and papillary dermis. Hematoxylin and eosin stain, original magnification $(A, C) \times 200,(B, D) \times 400$.

negative/Melan-A-positive immunoreactivity in the lining cells thereof. ${ }^{27}$ Additional immunohistochemical staining for CD31/Melan-A revealed three different patterns of immunoreactivity in the dermal cystic TI-LIOB lesions: 1) cystic cavities with CD31-positive/Melan-A-negative lining cells, 2) pseudo-cystic cavities with adjacent CD31positive/Melan-A-negative microvascular components, and 3) CD31-negative/Melan-A-negative pseudo-cystic cavitation without adjacent microvascular components. ${ }^{27}$ Moreover, CD31-negative/Melan-A-positive, epidermal TI-LIOB lesions were usually bigger in size, compared to CD31-positive/Melan-A-negative or CD31-negative/ Melan-A-negative dermal TI-LIOB lesions. ${ }^{27}$

An in vivo rat skin study indicated that both MLAand DOE-type fractionated optics during 1,064-nm picosecond-domain $\mathrm{Nd}$ :YAG laser treatments generate cystic vacuoles in the lower epidermis or upper papillary dermis. ${ }^{28}$ Therein, post-treatment Day 21 specimens exhibited mildly increased epidermal thickness and upper dermal collagen fibers. ${ }^{28}$ Additionally, picosecond lasertreated rat skin specimens, which were pre-treated with cohesive polydensified matrix hyaluronic acid (HA) in the subdermis, exhibited more remarkable increases in epidermal thickness and fibroblasts and collagen fibers in the papillary dermis at post-treatment Day 10 and 21, compared to HA-untreated, picosecond laser-treated rat skin. ${ }^{28}$ Immunohistochemical staining for cluster of differentiation 44 (CD44) additionally revealed that fractionated picosecond laser treatments stimulated up-regulated expression of CD44 in basilar epidermal cells and dermal fibroblasts. ${ }^{28}$ Thus, an HA-rich environment and up-regulated CD44 expression may enhance skin regeneration in fractional picosecond-domain laser-induced treatment.

\section{Plasma}

Plasma is generated from inert gaseous sources, including ambient air, argon, helium, and nitrogen and, in clinical applications, can emitted to target tissue at a pulse duration in the milliseconds. ${ }^{30-32}$ Therein, ultra-high frequency generators using RF or microwave energy are used to stripe electrons from atoms. ${ }^{30,31}$ Nitrogen gas is an inert diatomic molecule, and nitrogen plasma gener- 
ates predictable patterns of thermal damage and modification areas in the epidermis and dermis in a chromophore-independent manner. ${ }^{30-32}$ Central areas of nitrogen plasma-induced thermal tissue reactions exhibit irreversible cell damage, whereas the surrounding area of thermal modification shows reversible cell reactions. ${ }^{30,31}$

In an in vivo rat model study, researchers analyzed skin and skin appendageal reactions after atmosphericpressure, non-thermal, nitrogen-plasma pulse treatments at low-energy (0.5 J, $1.0 \mathrm{~J}, 1.5 \mathrm{~J}$, and $2.0 \mathrm{~J}$ ) and high-energy (2.5 J, $3.0 \mathrm{~J}, 3.5 \mathrm{~J}$, and $4.0 \mathrm{~J}$ ) settings (Fig. 5). ${ }^{32}$ Single-pulse nitrogen plasma-induced tissue reactions ranged from 1) no remarkable microscopic epidermal changes with mildly dilated dermal microvasculature and 2) mild to moderate eosinophilic epidermal reactions with microscopic vacuolar changes of epidermal cells and mildly dilated dermal microvasculature to 3 ) moderate to marked eosinophilic epidermal reactions without vacuolar changes of epidermal cells with moderately dilated dermal microvasculature by increasing pulse energy. ${ }^{32}$
Moreover, the single-pulse, nitrogen-plasma treatments resulted in immediate skin appendageal reactions of grey to black changes in the sebaceous glands with or without follicular epidermal vacuolization reactions at the experimental settings of 1.0-4.0 J. ${ }^{32}$ Rat skin specimens at Day 0 , which were treated with five consecutive passes of nitrogen plasma at low-energy settings, generated noticeable tissue coagulation at the depths of $31.5 \pm 8.3$ at $1.0 \mathrm{~J}, 94.9$ \pm 16.9 at $1.5 \mathrm{~J}$, and $171.6 \pm 19.7$ at $2.0 \mathrm{~J}$ over the epidermis and upper dermis. ${ }^{32}$ At Day 21 after treatment, the rat skin specimens exhibited well-demarcated, marked increases in upper dermal fibroblasts and collagen fibers at the depths of $79.7 \pm 8.6 \mu \mathrm{m}$ at $1.0 \mathrm{~J}, 98.7 \pm 14.0 \mu \mathrm{m}$ at $1.5 \mathrm{~J}$, and $134.6 \pm 10.8 \mathrm{\mu m}$ at $2.0 \mathrm{~J} \mathrm{~J}^{32}$ Meanwhile, Day-0 rat skin specimens treated with five consecutive passes of nitrogen plasma at high-energy settings showed noticeable tissue coagulation at the depths of $381.7 \pm 33.6$ at $2.5 \mathrm{~J}$ and $453.3 \pm 75.7 \mu \mathrm{m}$ at $3.0 \mathrm{~J}$. $^{32}$ The Day-21 rat skin specimens exhibited well-demarcated and significant increases in upper to mid dermal fibroblasts and collagen
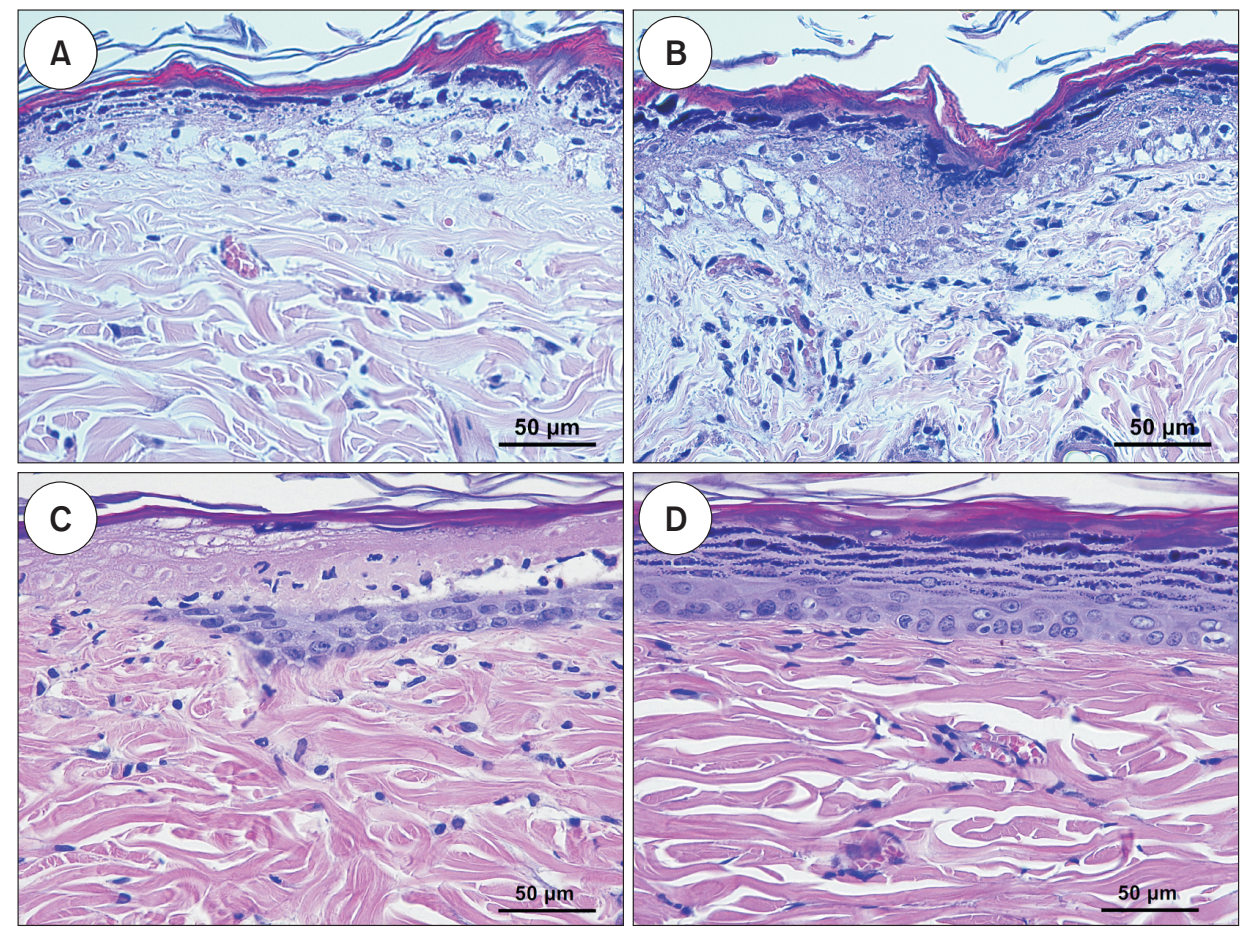

Fig. 5. In vivo rat skin reactions after argon plasma and nitrogen plasma treatments. (A, B) The in vivo male Sprague-Dawley rat skin was treated with 15 passes of microwave-generated, atmospheric-pressure, non-thermal argon plasma using a plasma generator (Pladuo ${ }^{\mathrm{TM}}$; Shenb Co., Ltd., Seoul, Korea) at the energy setting of $0.8 \mathrm{~J}$ and a distance setting of $5 \mathrm{~mm}$. Multiple passes of argon plasma treatment generates remarkable vacuolar changes in the entire epidermis and mild thermal tissue reactions with inflammatory cell infiltration in the upper dermis. Microscopic photographs were taken (A) immediately and (B) 1 day after argon plasma treatment. (C, D) In vivo rat skin treated with five passes of microwavegenerated, atmospheric-pressure, non-thermal nitrogen plasma treatment at the energy setting of $1.5 \mathrm{~J}$ and a distance setting of $5 \mathrm{~mm}$. Nitrogen plasma-induced thermal coagulation was found throughout the entire epidermis with microscopic features of partial re-epithelization at Day 1 and complete re-epithelization at Day 5 . Hematoxylin and eosin stain, original magnification $\times 400$. 
fibers at the depths of $395.7 \pm 23.0 \mu \mathrm{m}$ at $2.5 \mathrm{~J}$ and 846.7 $\pm 26.1 \mu \mathrm{m}$ at $3.0 \mathrm{~J}$, with scar formation in the absence of pilosebaceous units. ${ }^{32}$

Argon plasma has been used for various medical purposes due to its antibacterial, antiviral, antifungal, antipruritic, and skin renewal effects. ${ }^{7-9}$ An in vivo rat study using atmospheric-pressure, non-thermal, argonplasma pulses investigated argon plasma-induced tissue reactions at energy settings of $0.25 \mathrm{~J}, 0.35 \mathrm{~J}, 0.45 \mathrm{~J}, 0.6$ $\mathrm{J}, 0.75 \mathrm{~J}, 0.85 \mathrm{~J}, 1.1 \mathrm{~J}$, and $1.3 \mathrm{~J} .^{32} \mathrm{~A}$ single pulse of argon plasma induced tissue reactions of 1) no remarkable microscopic epidermal and dermal changes with mild cleavage lines in the dermo-epidermal junctions and 2) mild to moderate eosinophilic epidermal reactions with pyknotic nuclei and perinuclear vacuolization reactions in the basilar epidermis, depending on the pulse energy. ${ }^{32}$ Moreover, single-pulse argon-plasma treatments also elicited immediate thermal reactions in the sebaceous glands with or without follicular epidermal vacuolization. ${ }^{32}$ In the study, rat skin specimens at Day 0 that were treated with five consecutive passes of argon plasma at energy settings of $0.35,0.45,0.6,0.75$, and $0.85 \mathrm{~J}$ exhibited noticeable tissue reactions, including thinning of the epidermis, mild inflammatory cell infiltration in the upper dermis, and homogenous thermal reactions without remarkable desiccation in the sebaceous glands. ${ }^{32}$ Moreover, treatment with 15 pulses of argon plasma generated immediate skin reactions of necrotic, vacuolar histologic changes in the lower epidermis and follicular epidermis at $0.45 \mathrm{~J}$ or in the entire epidermis with upper dermal tissue coagulation and vascular congestion at $0.8 \mathrm{~J}$ or $1.3 \mathrm{~J} .{ }^{32} \mathrm{Ad}-$ ditionally, varying degrees of desiccative reactions in the sebaceous glands were also found..$^{32}$ The argon plasmainduced tissue reactions were more pronounced at Day 1 and Day 5 after treatment than at Day 0 , with greater degrees of epidermal and follicular epidermal vacuolization, along with pyknotic nuclei and upper dermal inflammatory cell infiltration and congested microvascular components. ${ }^{32}$ Although the plasma-induced epidermal and dermal reactions almost completely recovered within 7-10 days, rat skin specimens treated with 15 pulses of argon plasma at $1.3 \mathrm{~J}$ presented delayed epithelialization and dense inflammatory cell infiltration in the dermis with injured pilosebaceous units at Day $10 .{ }^{32}$

\section{HUMAN FIBROBLAST-DERIVED MULTI-PEPTIDE FACTORS}

The role of human fibroblasts during wound repair

Injured skin requires various types of cells, growth fac- tors, and cytokines for wound repair; however, the crucial factors that regulate skin regeneration have not been fully elucidated. Research has indicated that during wound repair, active cellular interactions among keratinocytes, neutrophils, endothelial cells, macrophages, and fibroblasts contribute to the regeneration of epidermal and dermal components. ${ }^{33}$ Vascular endothelial growth factor (VEGF), platelet-derived growth factor (PDGF), epidermal growth factor, keratinocyte growth factor (KGF), hepatocyte growth factor (HGF), interleukin (IL), transforming growth factor (TGF)- $\beta 1$, and matrix metalloproteinases are also known play key roles during wound repair. ${ }^{33}$

The process of wound repair encompasses five distinctive phases: the hemostatic phase, inflammatory phase, granulation and angiogenic phase, re-epithelialization phase, and tissue remodeling phase, in that order. ${ }^{33}$ Phase changes are managed through cell to cell communication, and signaling molecules act as a communicator between cells. Various types of cells produce signaling molecules as a modulator, and these modulators include growth factors, cytokines, and chemokines.

The homeostatic phase involve the aggregation of platelets to form blood clots to prevent excessive bleeding from the tissue damage. Blood clot formation creates a matrix for cells to migrate and initiates the inflammatory phase. ${ }^{33}$ During inflammation, immune cells are recruited to defend against infectious agents and to induce growth factor production, such as TGF- $\beta 1$, VEGF, and PDGF. ${ }^{33}$ TGF- $\beta 1$ stimulates cells to synthesize extracellular matrix components; PDGF promotes chemotaxis for migrating neutrophils, monocytes, and fibroblasts and enhances fibroblast proliferation; and VEGF increases vascular permeability and enhances inflammatory cell infiltration. ${ }^{33}$ During the proliferation phase, granulation tissue is created by producing collagen matrix and blood vessels. Therein, TGF- $\beta 1$ stimulates fibroblasts to proliferate, VEGF migrates and proliferates endothelial cells for blood vessel formation, and PDGF enhances fibroblast proliferation and extracellular matrix production. ${ }^{33}$

The re-epithelialization phase initiates the recovery of the epidermis by stimulating the proliferation of keratinocytes. To do so, KGF and basic fibroblast growth factor (bFGF) bind to receptors in the keratinocytes and endothelial cells to proliferate and migrate for reepithelialization. ${ }^{33}$ The last phase of wound repair is tissue remodeling. ${ }^{33}$ Tissue remodeling occurs to strengthen wound tensile by replacing collagen type 3 with type 1 . Fibroblast and TGF- $\beta 1$ coordinate to complete the last step of the wound healing process. ${ }^{33}$ Complex epithelialmesenchymal interactions during wound repair also ac- 


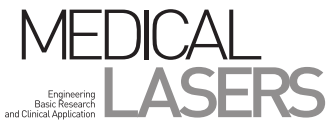

celerate rapid wound closure and reduce pathologic collagen production via matricellular protein angiopoietinlike 4 and IL-1. ${ }^{34,35}$

Among the cellular components, dermal fibroblasts are responsible not only for forming matrix proteins and collagen fibrils in the dermis, but also for promoting epidermal growth. ${ }^{36-38}$ Dermal fibroblast populations exhibit distinct properties depending on the dermal microenvironment, aging process, and species. ${ }^{36,39}$ Previous studies have demonstrated that patterns of growth factor and cytokine secretion differ between site-matched papillary and reticular human dermal fibroblasts. ${ }^{38,40}$ Therein, papillary fibroblasts more effectively interact with epidermal cells to promote keratinocyte proliferation and differentiation, compared with reticular fibroblasts. ${ }^{40}$ Furthermore, the differentiation of papillary fibroblasts to reticular fibroblasts can be induced by aging processes or TGF- $\beta$ 1 stimulation. ${ }^{41,42}$ In aged skin, the therapeutic restoration of dermal extracellular matrix activates fibroblasts, endothelial cells, and keratinocytes to enhance functional skin rejuvenation. ${ }^{39}$

\section{The composition of human fibroblast-derived multi-peptide factors}

Multi-peptide factors (MPFs), which are secreted from dermal fibroblasts, have been suggested to play crucial roles during wound repair. ${ }^{33,40}$ Researchers have analyzed fibroblast-derived MPFs by evaluating growth factors and chemoattractive factors therewithin using the culture medium of allogeneic dermal fibroblasts. ${ }^{43}$ Therein, fresh allogeneic cultured dermal substitute (CDS) containing fibroblasts were found to release VEGF, bFGF, HGF, PDGF-AA, TGF- $\beta 1, K G F, I L-6$, and IL-8. ${ }^{43}$ These growth factors and chemoattractive factors activate and recruit fibroblasts and endothelial cells and promote extracellular matrix deposition, including hyaluronic acid, collagen, fibronectin, and protease inhibitor. Important for clinical purpose, the re-cultivation of cryopreserved CDS after thawing thereof was found to release the same amounts of VEGF, bFGF, and HGF, which are essential for wound repair, as fresh CDS. ${ }^{43}$ The levels of PDGF-AA, TGF- $\beta 1$, $K G F$, IL-6, and IL-8, however, were higher in fresh CDS than in cryopreserved CDS. ${ }^{43}$

In addition to CDS containing fibroblasts, dermal fibroblast-conditioned medium (DFCM) has also been shown to contain MPFs. ${ }^{44,45}$ During in vitro expansion of human keratinocytes, the supplementation of DFCM significantly increased the proliferation of keratinocytes and the efficiency of keratinocyte attachment. ${ }^{44}$ Moreover, in vitro wound the healing rate of keratinocytes was significantly higher when using serum-free keratinocyte culture medium supplemented with DFCM at $10 \mu \mathrm{g} / \mathrm{ml}$, compared with control, DFCM at $20 \mathrm{\mu g} / \mathrm{ml}$, and DFCM at $40 \mu \mathrm{g} /$ $\mathrm{ml}{ }^{45}$ Proteomic analyses of DFCM revealed that DFCM contained serum albumin, lactotransferrin, fibronectin, thrombospondin-1, pentraxin-related protein PTX3, collagen alpha-1 (VI) chain, decorin, alpha-2-macroglobin, pregnancy zone protein, inter-alpha-trypsin inhibitor heavy chain $\mathrm{H} 2$, fibulin-1, collagen alpha-1 (I) chain, nucleobindin-1, pigment epithelium-derived factor, alphafetoprotein, collagen alpha-1 (XII) chain, alpha-2-HS-glycoprotein, and transforming growth factor-beta-induced protein ig-h3. ${ }^{46}$ These human dermal fibroblast secreting proteins are involved in cell adhesion, attachment, proliferation, and migration to promote wound repair. ${ }^{46}$

\section{The role of human fibroblast-derived MPFs dur- ing the use of energy-delivering systems}

As stated above, fibroblasts from different species or anatomical sites exhibit distinct transcriptional properties with high heterogeneity, and these fibroblast subpopulations contribute to different aspects of cutaneous development and homeostasis. Accordingly, as various in vitro experimental conditions, including serum-free starvation or other stimulations, how different patterns of MPF secretion can be different, the use of allogeneic fibroblasts and proper cultivation settings are necessary to obtain MPFs that can enhance epithelial-mesenchymal interactions during wound repair.

Human fibroblast-derived MPFs have been used during treatments with energy-delivering modalities to enhance energy-induced tissue reactions or to assist in delivering therapeutic drugs to targeted layers of the skin (Fig. 6). Although topical application is a simple and safe mode of drug delivery, the bioavailability thereof is significantly limited by the stratum corneum. ${ }^{50}$ Ablative fractional laser and invasive RF treatments can enhance the penetration of active substances, including human fibroblast-derived MPFs, which are applied immediately thereafter, by disrupting the integrity of the stratum corneum. ${ }^{50-52}$ Previous studies have demonstrated that deeper penetration of fractional ablative zones that extends into the dermis does not further improve the bioavailability of topically applied agents, however ${ }^{50-52}$ Furthermore, non-ablative energy sources also have been suggested to improve the penetration of applied agents by temporarily expanding intercellular spaces of the stratum corneum via photomechanical and/or photothermal tissue reactions. ${ }^{50,53}$ Accordingly, human fibroblast-derived MPFs are viable for topical application immediately after treatments with 

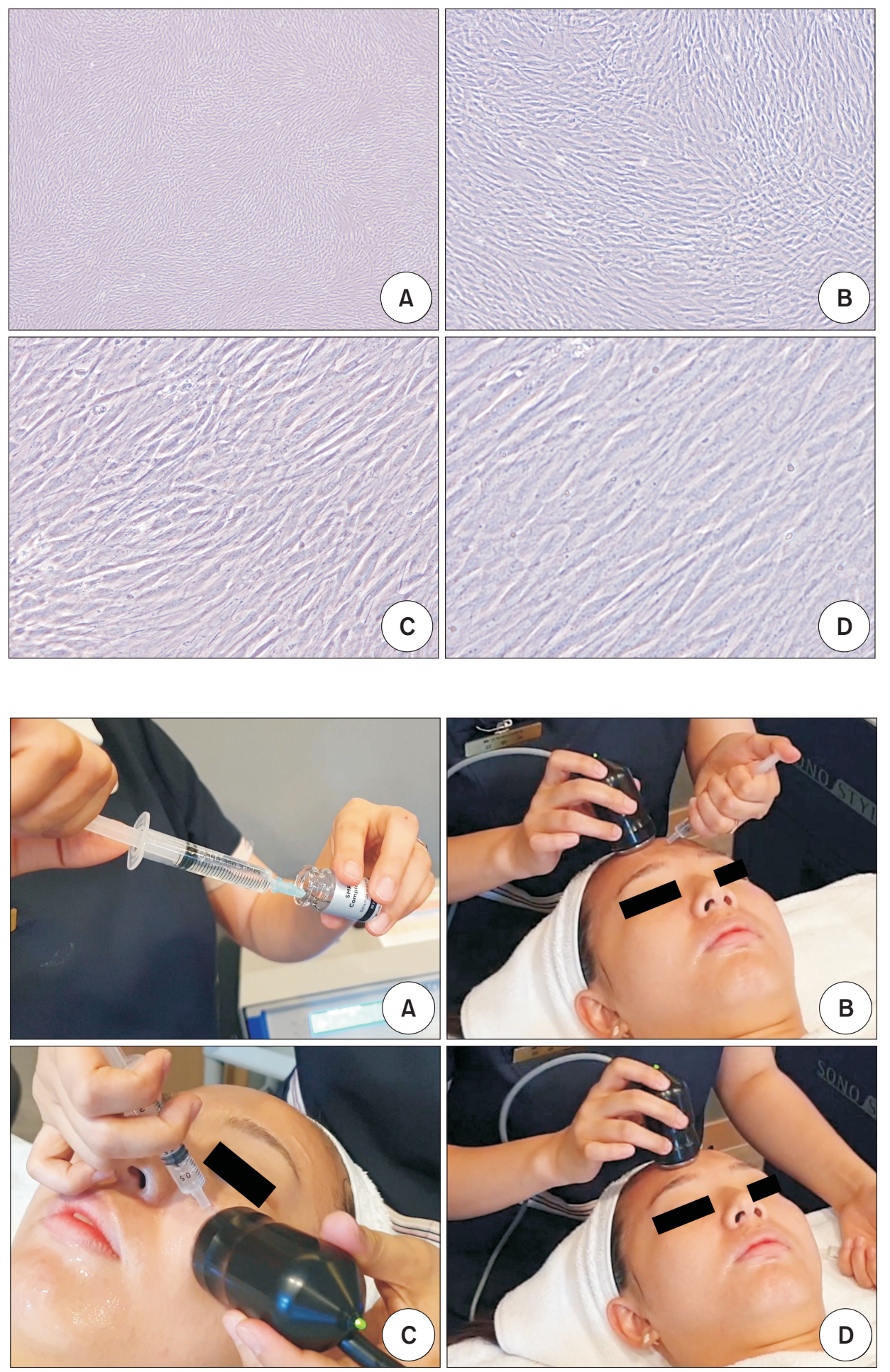

Fig. 6. Optical microscopic, phasecontrast images of cultured human fibroblasts. Normal neonatal human dermal fibroblasts were purchased (CC-2511; Lonza Inc., Rockland, ME, USA) and cultured as a monolayer. Numerous, homogenous spindle-shaped dermal fibroblasts were obtained, and dermal fibroblast-conditioned medium contained human fibroblast-derived multi-peptide factors (MPFs). Unstained, original magnification $(\mathrm{A}) \times 40,(\mathrm{~B}) \times 100$, (C) $\times 200$, (D) $\times 400$.
Fig. 7. Post-treatment application of human fibroblast-derived MPFs. (A) Human fibroblast-derived MPFs $\left(\mathrm{SMPF}^{\circledR}\right.$ complex; BNV Biolab, Seoul, Korea) are prepared using a sterile syringe. (B-D) Immediately after treatment using an energydelivering device, human fibroblastderived MPFs are applied on the entire face along with ultrasound (Sono Styler ${ }^{\circledR}$; Weyergans, Dueren, Germany) treatment, which enhances the penetration of MPFs into the skin. various ablative or non-ablative, invasive or non-invasive energy-delivering devices, depending on the therapeutic purposes and skin condition (Fig. 7).

In common pigmentation disorders in Asian patients, including senile lentigo and melasma, senescent fibroblasts and endothelial cells have been shown to secrete crucial factors that significantly upregulate epidermal melanogenesis. ${ }^{54,55}$ While conventional therapeutic modalities for pigmentary disorders mainly target melanocytes and melanin chromophores in the epidermis and/or dermis, recent modalities have been designed to additionally treat senescent fibroblasts and endothelial cells in 
the dermis. ${ }^{54-56}$ Energy-delivering modalities that directly affect senescent fibroblasts and endothelial cells in pigmentary lesions can include NAFL, AFL, non-invasive or invasive RF, picosecond-domain lasers, and atmosphericpressure, non-thermal nitrogen plasma. ${ }^{54-56}$ However, Asian patients pose a high risk of post-inflammatory hyperpigmentation and worsening of lentiginous or melasma lesions. Thus, the use of human fibroblast-derived MPFs in combination with energy-delivering devices at safe treatment parameter ranges has been suggested to promote post-treatment wound repair and reduce the risk of side effects. Moreover, human fibroblast-derived MPFs could theoretically induce epithelial recovery and homeostasis over the senescent fibroblast-secreted pathologic factors.

\section{CONCLUSIONS}

Human fibroblasts secrete MPFs, including VEGF, bFGF, HGF, PDGF-AA, TGF- $\beta 1$, KGF, IL-6, and IL-8, that activate and recruit fibroblasts and endothelial cells and promote re-epithelialization and extracellular matrix deposition. Human fibroblast-derived MPFs have been used during treatments with energy-delivering modalities to improve the penetration of MPFs into the skin and to enhance energy-induced tissue reactions. Depending on the therapeutic goal, energy-delivering devices should be selected according to the efficacy and safety of the energy source on the pathologic skin condition and the major target skin layers.

\section{ACKNOWLEDGEMENTS}

We would like to thank Sung Hun Suh (BNV Biolab, Seoul, Korea), Sunny Kang (Shenb Co., Ltd., Seoul, Korea), Bora Kim (Shenb Co., Ltd.), Min Choi (Shenb Co., Ltd.), Jinyoung Park (Lutronic Corp., Goyang, Korea), and Herin Lyu (Lutronic Corp.) for their assistance with technical support. We would also like to thank Anthony Thomas Milliken, ELS (Editing Synthase, Seoul, Korea) for his help with the editing of this manuscript. This research was supported by the 2019 scientific promotion program funded by Jeju National University.

\section{CONFLICTS OF INTEREST}

The authors declare no conflicts of interest.

\section{FUNDING}

This study was supported by research funding from BNV Biolab. The funding company had no role in the study design, data collection, data analysis, manuscript preparation, or publication. The authors have indicated no significant interest with commercial supporters.

\section{REFERENCES}

1. Karmisholt KE, Wenande E, Thaysen-Petersen D, Philipsen PA, Paasch U, Haedersdal M. Early intervention with non-ablative fractional laser to improve cutaneous scarring-A randomized controlled trial on the impact of intervention time and fluence levels. Lasers Surg Med 2018;50:28-36.

2. Cho SB, Lee JH, Choi MJ, Lee KY, Oh SH. Efficacy of the fractional photothermolysis system with dynamic operating mode on acne scars and enlarged facial pores. Dermatol Surg 2009;35:108-14.

3. Cho SB, Goo BL, Zheng Z, Yoo KH, Kang JS, Kim H. Therapeutic efficacy and safety of a 1927-nm fractionated thulium laser on pattern hair loss: an evaluator-blinded, split-scalp study. Lasers Med Sci 2018;33:851-9.

4. Stewart N, Lim AC, Lowe PM, Goodman G. Lasers and laserlike devices: part one. Australas J Dermatol 2013;54:173-83.

5. Orringer JS, Rittié L, Hamilton T, Karimipour DJ, Voorhees JJ, Fisher GJ. Intraepidermal erbium: YAG laser resurfacing: impact on the dermal matrix. J Am Acad Dermatol 2011;64:11928.

6. Cho SB, Kim JS, Zheng Z, Choi MJ, Choi IG, Oh HS, et al. Decreased tissue and serum expression of galectin-7 in patients with hypertrophic scars. Acta Derm Venereol 2013;93:669-73.

7. Chu KF, Dupuy DE. Thermal ablation of tumours: biological mechanisms and advances in therapy. Nat Rev Cancer 2014;14:199-208.

8. White WM, Makin IR, Slayton MH, Barthe PG, Gliklich R. Selective transcutaneous delivery of energy to porcine soft tissues using Intense Ultrasound (IUS). Lasers Surg Med 2008;40:6775.

9. Kim HJ, Kim HG, Zheng Z, Park HJ, Yoon JH, Oh W, et al. Coagulation and ablation patterns of high-intensity focused ultrasound on a tissue-mimicking phantom and cadaveric skin. Lasers Med Sci 2015;30:2251-8.

10. Kim H, Ahn KJ, Lee S, Park H, Cho SB. Interactive thermal tissue reactions of 7-MHz intense focused ultrasound and $1-\mathrm{MHz}$ and 6-MHz radiofrequency on cadaveric skin. Skin Res Technol 2019;25:171-8.

11. Suh DH, Choi JH, Lee SJ, Jeong KH, Song KY, Shin MK. Comparative histometric analysis of the effects of high-intensity focused ultrasound and radiofrequency on skin. J Cosmet Laser 
Ther 2015;17:230-6.

12. Na J, Zheng Z, Dannaker C, Lee SE, Kang JS, Cho SB. Electromagnetic initiation and propagation of bipolar radiofrequency tissue reactions via invasive non-insulated microneedle electrodes. Sci Rep 2015;5:16735.

13. Zheng Z, Goo B, Kim DY, Kang JS, Cho SB. Histometric analysis of skin-radiofrequency interaction using a fractionated microneedle delivery system. Dermatol Surg 2014:40:134-41.

14. Taheri A, Mansoori P, Sandoval LF, Feldman SR, Williford PM, Pearce D. Entrance and propagation pattern of high-frequency electrical currents in biological tissues as applied to fractional skin rejuvenation using penetrating electrodes. Skin Res Technol 2014;20:270-3.

15. Taheri A, Mansoori P, Sandoval LF, Feldman SR, Pearce D, Williford PM. Electrosurgery: part I. Basics and principles. J Am Acad Dermatol 2014;70:591.e1-591.e14.

16. Taheri A, Mansoori P, Sandoval LF, Feldman SR, Pearce D, Williford PM. Electrosurgery: part II. Technology, applications, and safety of electrosurgical devices. J Am Acad Dermatol 2014;70:607.e1-607.e12.

17. Nelson AA, Beynet D, Lask GP. A novel non-invasive radiofrequency dermal heating device for skin tightening of the face and neck. J Cosmet Laser Ther 2015;17:307-12.

18. Boisnic S, Divaris M, Branchet MC, Nelson AA. Split-face histological and biochemical evaluation of tightening efficacy using temperature- and impedance-controlled continuous non-invasive radiofrequency energy. J Cosmet Laser Ther 2017;19:12832.

19. Boisnic S, Divaris M, Nelson AA, Gharavi NM, Lask GP. A clinical and biological evaluation of a novel, noninvasive radiofrequency device for the long-term reduction of adipose tissue. Lasers Surg Med 2014;46:94-103.

20. Mulholland RS, Paul MD, Chalfoun C. Noninvasive body contouring with radiofrequency, ultrasound, cryolipolysis, and lowlevel laser therapy. Clin Plast Surg 2011;38:503-20, vii-iii.

21. Mulholland RS. Radio frequency energy for non-invasive and minimally invasive skin tightening. Clin Plast Surg 2011;38:43748, vi.

22. Zhang B, Moser MAJ, Zhang EM, Luo Y, Zhang W. A new approach to feedback control of radiofrequency ablation systems for large coagulation zones. Int J Hyperthermia 2017;33:36777.

23. Bernstein EF, Schomacker KT, Basilavecchio LD, Plugis JM, Bhawalkar JD. Treatment of acne scarring with a novel fractionated, dual-wavelength, picosecond-domain laser incorporating a novel holographic beam-splitter. Lasers Surg Med 2017:49:796-802.

24. Varghese B, Bonito V, Jurna M, Palero J, Verhagen MH. Influence of absorption induced thermal initiation pathway on irradiance threshold for laser induced breakdown. Biomed Opt
Express 2015;6:1234-40.

25. Ahn KJ, Zheng Z, Kwon TR, Kim BJ, Lee HS, Cho SB. Pattern analysis of laser-tattoo interactions for picosecond- and nanosecond-domain 1,064-nm neodymium-doped yttriumaluminum-garnet lasers in tissue-mimicking phantom. Sci Rep 2017;7:1533.

26. Lee HC, Childs J, Chung HJ, Park J, Hong J, Cho SB. Pattern analysis of 532- and 1,064-nm picosecond-domain laserinduced immediate tissue reactions in ex vivo pigmented micropig skin. Sci Rep 2019;9:4186.

27. Chung HJ, Lee HC, Park J, Childs J, Hong J, Kim H, et al. Pattern analysis of 532- and 1064-nm microlens array-type, picosecond-domain laser-induced tissue reactions in ex vivo human skin. Lasers Med Sci 2019;34:1207-15.

28. Kim HK, Kim HJ, Hong JY, Park J, Lee HC, Lyu H, et al. Interactive tissue reactions of $1064-\mathrm{nm}$ focused picosecond-domain laser and dermal cohesive polydensified matrix hyaluronic acid treatment in in vivo rat skin. Skin Res Technol. In press 2020.

29. Meran S, Luo DD, Simpson R, Martin J, Wells A, Steadman R, et al. Hyaluronan facilitates transforming growth factor- $\beta 1$-dependent proliferation via CD44 and epidermal growth factor receptor interaction. J Biol Chem 2011;286:17618-30.

30. Kilmer S, Semchyshyn N, Shah G, Fitzpatrick R. A pilot study on the use of a plasma skin regeneration device (Portrait PSR3) in full facial rejuvenation procedures. Lasers Med Sci 2007;22:101-9.

31. Elsaie ML, Kammer JN. Evaluation of plasma skin regeneration technology for cutaneous remodeling. J Cosmet Dermatol 2008; 7:309-11.

32. Kim H, Kim HJ, Kim HK, Hong JY, Cho SB. Effects of argon and nitrogen plasma pulses on the skin and skin appendages in an in vivo animal model. Skin Res Technol 2020;26:81-90.

33. Gurtner GC, Werner S, Barrandon Y, Longaker MT. Wound repair and regeneration. Nature 2008;453:314-21.

34. Teo Z, Chan JSK, Chong HC, Sng MK, Choo CC, Phua GZM, et al. Angiopoietin-like 4 induces a $\beta$-catenin-mediated upregulation of ID3 in fibroblasts to reduce scar collagen expression. Sci Rep 2017;7:6303.

35. Chong HC, Tan MJ, Philippe V, Tan SH, Tan CK, Ku CW, et al. Regulation of epithelial-mesenchymal IL-1 signaling by PPARbeta/delta is essential for skin homeostasis and wound healing. J Cell Biol 2009;184:817-31.

36. Lawlor KT, Kaur P. Dermal contributions to human interfollicular epidermal architecture and self-renewal. Int J Mol Sci 2015;16:28098-107.

37. Leary T, Jones PL, Appleby M, Blight A, Parkinson K, Stanley M. Epidermal keratinocyte self-renewal is dependent upon dermal integrity. J Invest Dermatol 1992;99:422-30.

38. Tuan TL, Keller LC, Sun D, Nimni ME, Cheung D. Dermal fibroblasts activate keratinocyte outgrowth on collagen gels. J Cell 
Sci 1994; 107(Pt 8):2285-9.

39. Quan T, Wang F, Shao Y, Rittié L, Xia W, Orringer JS, et al. Enhancing structural support of the dermal microenvironment activates fibroblasts, endothelial cells, and keratinocytes in aged human skin in vivo. J Invest Dermatol 2013;133:658-67.

40. Sorrell JM, Baber MA, Caplan Al. Site-matched papillary and reticular human dermal fibroblasts differ in their release of specific growth factors/cytokines and in their interaction with keratinocytes. J Cell Physiol 2004;200:134-45.

41. Janson D, Saintigny G, Mahé C, El Ghalbzouri A. Papillary fibroblasts differentiate into reticular fibroblasts after prolonged in vitro culture. Exp Dermatol 2013;22:48-53.

42. Janson D, Saintigny G, Zeypveld J, Mahé C, El Ghalbzouri A. TGF- $\beta 1$ induces differentiation of papillary fibroblasts to reticular fibroblasts in monolayer culture but not in human skin equivalents. Eur J Dermatol 2014;24:342-8.

43. Kubo K, Kuroyanagi Y. A study of cytokines released from fibroblasts in cultured dermal substitute. Artif Organs 2005;29:8459.

44. Chowdhury SR, Aminuddin BS, Ruszymah BH. Effect of supplementation of dermal fibroblasts conditioned medium on expansion of keratinocytes through enhancing attachment. Indian J Exp Biol 2012;50:332-9.

45. Manira M, Chowdhury SR, Rosliza A, Yi Ling A, Abidah A, Vittarino J, et al. Concentration dependent effect of dermal fibroblast conditioned medium on in vitro wound healing properties of keratinocytes. Regen Res 2014;3:30-2.

46. Maarof M, Lokanathan Y, Ruszymah HI, Saim A, Chowdhury SR. Proteomic analysis of human dermal fibroblast conditioned medium (DFCM). Protein J 2018;37:589-607.

47. Ohtani T, Okamoto K, Kaminaka C, Kishi T, Sakurane M, Yamamoto $Y$, et al. Digital gangrene associated with idiopathic hypereosinophilia: treatment with allogeneic cultured dermal substitute (CDS). Eur J Dermatol 2004;14:168-71.

48. Moroi Y, Fujita S, Fukagawa S, Mashino T, Goto T, Masuda T et al. Clinical evaluation of allogeneic cultured dermal substitutes for intractable skin ulcers after tumor resection. Eur J Derma- tol 2004;14:172-6.

49. Hasegawa T, Suga Y, Mizoguchi M, Ikeda S, Ogawa H, Kubo K, et al. Clinical trial of allogeneic cultured dermal substitute for the treatment of intractable skin ulcers in 3 patients with recessive dystrophic epidermolysis bullosa. J Am Acad Dermatol 2004;50:803-4.

50. Braun SA, Schrumpf H, Buhren BA, Homey B, Gerber PA. Laser-assisted drug delivery: mode of action and use in daily clinical practice. J Dtsch Dermatol Ges 2016;14:480-8.

51. Haak CS, Farinelli WA, Tam J, Doukas AG, Anderson RR, Haedersdal M. Fractional laser-assisted delivery of methyl aminolevulinate: Impact of laser channel depth and incubation time. Lasers Surg Med 2012;44:787-95.

52. Lee WR, Shen SC, Pai MH, Yang HH, Yuan CY, Fang JY. Fractional laser as a tool to enhance the skin permeation of 5-aminolevulinic acid with minimal skin disruption: a comparison with conventional erbium:YAG laser. J Control Release 2010;145:124-33.

53. Lim HK, Jeong KH, Kim NI, Shin MK. Nonablative fractional laser as a tool to facilitate skin penetration of 5 -aminolaevulinic acid with minimal skin disruption: a preliminary study. $\mathrm{Br} \mathrm{J}$ Dermatol 2014;170:1336-40.

54. Kim M, Kim SM, Kwon S, Park TJ, Kang HY. Senescent fibroblasts in melasma pathophysiology. Exp Dermatol 2019;28:71922.

55. Yoon JE, Kim Y, Kwon S, Kim M, Kim YH, Kim JH, et al. Senescent fibroblasts drive ageing pigmentation: A potential therapeutic target for senile lentigo. Theranostics 2018;8:4620-32.

56. Kim HJ, Kim H, Kim YK, Cho SB. Treatment of refractory melasma with microwave-generated, atmospheric-pressure, nonthermal nitrogen plasma. Med Laser 2019;8:74-9.

How to cite this article: Suh SB, Ahn KJ, Chung HJ, Suh JY, Cho SB. Human fibroblast-derived multi-peptide factors and the use of energy-delivering devices in asian patients. Med Laser 2020;9:12-24. https://doi.org/10.25289/ML.2020.9.1.12 